\title{
Dynamic response of multiwall boron nitride nanotubes subjected to impact
}

\author{
S T TALEBIAN ${ }^{1, *}$ and M TAHANI ${ }^{2}$ \\ ${ }^{1}$ Department of Mechanical Engineering, Faculty of Engineering, Neyshabur Branch, Islamic Azad University, \\ 9319797139 Neyshabur, Iran \\ ${ }^{2}$ Department of Mechanical Engineering, Faculty of Engineering, Ferdowsi University of Mashhad, \\ 91775-1111 Mashhad, Iran
}

MS received 6 August 2014; accepted 17 August 2015

\begin{abstract}
Dynamic behaviours of multiwall boron nitride nanotubes (MWBNNTs) with finite length were studied by employing continuum structure. Multiple elastic shells with nonlinear model of van der Waals interactions were used for developing an inclusive and inexpensive dynamical model of MWBNNTs. The systems of coupled partial differential equations were solved by applying the finite element method. Whole or part of thin and bulky MWBNNTs were exposed to external pressure impact and dynamical treatment were investigated and precision of results were checked by evaluating the answers with those found by other methods. Both deflection and stress studies were carried out for tubes of MWBNNTs and the influences of longitudinal wave caused by external pressure impact were considered in MWBNNTs. Also, time history diagrams of MWBNNTs with impact of initial longitudinal deflection were investigated and the extension of the longitudinal waves through the length of layers were shown and so, wave speed was determined.
\end{abstract}

Keywords. Multiwall boron nitride nanotube; dynamic response; impact; wave propagation.

\section{Introduction}

Boron nitride nanotubes (BNNTs) are like carbon nanotubes (CNTs) in structure in which carbon atoms are replaced by alternate boron and nitrogen atoms. Thus, BNNTs demonstrate extraordinary mechanical behaviours comparable to CNTs regarding to elevated modulus of elasticity and excellent stableness. Despite, BNNTs are dissimilar from CNTs in other sides such as high-temperature rigidity to oxidation. Their structural, mechanical, electronic, vibrational and optical attributes have been studied experimentally and theoretically. The moduli of elasticity of BNNTs with functional faults are studied. ${ }^{1}$ Also, based on the molecular mechanic simulations, an analytical 'stick-spiral' model of singlewall boron nitride nanotubes (SWBNNTs) have been demonstrated to study their elastic properties. ${ }^{2}$ In addition, the elastic properties of BNNTs are calculated by using the stress-deformation treatment found from the continuum lattice thermodynamic model. ${ }^{3}$ Further, the moduli of elasticity of doublewall boron nitride nanotubes (DWBNNTs) are studied by employing ab-initio density functional theory. ${ }^{4}$ Thermal conductivity and longitudinal tension of SWBNNTs are estimated through the molecular dynamics simulation. ${ }^{5}$

\footnotetext{
*Author for correspondence (taha_talebian@yahoo.com)
}

Further, some works on thermoelectro-mechanical treatment of BNNTs are studied. On the basis of strain gradient theory, electro-thermal dynamic behaviour of DWBNNTs are studied that are mated by visco-Pasternak media. ${ }^{6}$ Also, the influence of partly filled polyethylene foam core on the treatment of torsional buckling of polymeric cylinder reinforced by DWBNNTs are examined, and exposed to thermoelectro-mechanical loading by using energy technique. ${ }^{7}$ In addition, longitudinal buckling behaviour of DWBNNTs embedded in an elastic media is demonstrated under thermoelectro-mechanical loads. ${ }^{8}$ As well, nonlinear dynamic behaviours of a smart composite micro-tube prepared of poly-vinylidene fluoride reinforced by BNNTs are studied under electrothermal loading. ${ }^{9}$ Finally, thermoelectro-torsional buckling reaction of a DWBNNT is studied on the basis of nonlocal elasticity and piezoelasticity theories. $^{10}$

Dynamic response of CNTs is considered by many investigators ${ }^{11-14}$ but it is in preliminary studies for BNNTs. Dynamic behaviour of cantilevered SWBNNTs are represented, employing molecular dynamics-based 3-D atomistic analyser for intermediate landing situation of inserted mass. ${ }^{15}$ Also, a macroscopic continuum simulation is suggested to compute the long-wavelength optical phonons of SWBNNTs. ${ }^{16}$ In addition, the nonlinear vibration of the embedded DWBNNTs conveying viscous fluid is studied on the basis of nonlocal piezoelasticity. ${ }^{17}$ Further, the nonlocal axial and transverse oscillations of BNNT exposed to 
a moving nanoparticle are investigated using the piezoelastic method and surface stress on the basis of EulerBernoulli beam. ${ }^{18}$ Besides, thermoelectro-nonlinear dynamic behaviour of DWBNNTs conveying viscous fluid is analysed on the basis of nonlocal piezoelasticity and Euler-Bernoulli beam theory. ${ }^{19}$ Also, the influence of time discretization on the nonlinear transverse oscillations of SWBNNT carrying viscous fluid is found to be on the nonlocal piezoelasticity. ${ }^{20}$ Finally, vibrational properties of SWBNNT are investigated regarding point defects to apply SWBNNTs as nanosensors. ${ }^{21}$

To the extent of the author's knowledge, no work has been described for analysing MWBNNTs (larger than doublewall) exposed to impact in time domain. Thus the aim of the study is to develop a comprehensive continuum model suitable for the study of dynamical behaviour of MWBNNTs, although the developed model has some drawbacks compared with the already available models. Firstly, with the present model with continuum approach, the effects of molecular defects are not measured while they can be observed by molecular approach such as dynamic molecular modelling. Secondly, this model is two dimensional in radial and longitudinal direction, and the effects of variations in angular direction are not considered which will be looked at in the subsequent studies.

\section{The multiple elastic shell model}

Here, a MWBNNT is simulated with the multiple elastic shells as presented in figure 1. A polar coordinate system is introduced that the origin settled on the centre of one edge of MWBNNT and coordinates $r, \theta$ and $z$ are in the radial, peripheral and longitudinal directions, respectively (see figure 1). The axisymmetric equation of motion is ${ }^{22}$

$$
\begin{aligned}
& \frac{1}{r} \frac{\partial\left(r \sigma_{r}\right)}{\partial r}-\frac{\sigma_{\theta}}{r}+\frac{\partial \sigma_{r z}}{\partial z}=\rho \frac{\partial^{2} u_{r}}{\partial t^{2}}, \\
& \frac{1}{r} \frac{\partial\left(r \sigma_{r z}\right)}{\partial r}+\frac{\partial \sigma_{z}}{\partial z}=\rho \frac{\partial^{2} u_{z}}{\partial t^{2}},
\end{aligned}
$$

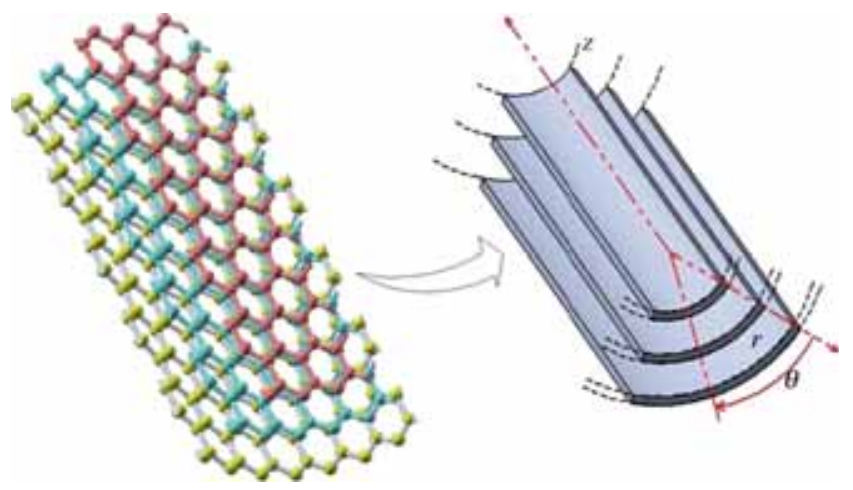

Figure 1. Section of the continuum model of a MWBNNT. where $u_{z}$ and $u_{r}$ indicate the deflection factors in the longitudinal and radial directions, respectively. Also, the straindisplacement rule is

$$
\varepsilon_{r}=\frac{\partial u_{r}}{\partial r}, \quad \varepsilon_{\theta}=\frac{u_{r}}{r}, \quad \varepsilon_{z}=\frac{\partial u_{z}}{\partial z}, \gamma_{r z}=\frac{\partial u_{z}}{\partial r}+\frac{\partial u_{r}}{\partial z} .
$$

Now, every tube of BNNT is simulated as an elastic isotropic thin shell. Hence, the constitutive relation is expressed as

$$
\left\{\begin{array}{c}
\sigma_{z} \\
\sigma_{\theta} \\
\sigma_{r}
\end{array}\right\}=\left[\begin{array}{lll}
C_{11} & C_{12} & C_{12} \\
C_{12} & C_{11} & C_{12} \\
C_{12} & C_{12} & C_{11}
\end{array}\right]\left\{\begin{array}{c}
\varepsilon_{z} \\
\varepsilon_{\theta} \\
\varepsilon_{r}
\end{array}\right\}, \sigma_{r z}=C_{66} \gamma_{r z},
$$

where

$$
\begin{aligned}
& C_{11}=\frac{E(1-v)}{(1+v)(1-2 v)}, \quad C_{12}=\frac{E v}{(1+v)(1-2 v)}, \\
& C_{66}=\frac{C_{11}-C_{12}}{2} .
\end{aligned}
$$

Upon replacement of equation (2) into equation (3), the constitutive relation can be composed as follows:

$$
\begin{aligned}
& \sigma_{z}=C_{11} \frac{\partial u_{z}}{\partial z}+C_{12}\left(\frac{u_{r}}{r}+\frac{\partial u_{r}}{\partial r}\right), \\
& \sigma_{\theta}=C_{11} \frac{u_{r}}{r}+C_{12}\left(\frac{\partial u_{z}}{\partial z}+\frac{\partial u_{r}}{\partial r}\right), \\
& \sigma_{r}=C_{11} \frac{\partial u_{r}}{\partial r}+C_{12}\left(\frac{u_{r}}{r}+\frac{\partial u_{z}}{\partial z}\right), \\
& \sigma_{r z}=C_{66}\left(\frac{\partial u_{z}}{\partial r}+\frac{\partial u_{r}}{\partial z}\right) .
\end{aligned}
$$

Substituting equation (5) into equation (1) gives the governing equation of motion:

$$
\begin{gathered}
\frac{1}{r} \frac{\partial}{\partial r}\left[C_{11} r \frac{\partial u_{r}}{\partial r}+C_{12}\left(u_{r}+r \frac{\partial u_{z}}{\partial z}\right)\right]+\frac{\partial}{\partial z}\left[C_{66}\left(\frac{\partial u_{z}}{\partial r}+\frac{\partial u_{r}}{\partial z}\right)\right] \\
-C_{11} \frac{u_{r}}{r^{2}}-\frac{C_{12}}{r}\left(\frac{\partial u_{r}}{\partial r}+\frac{\partial u_{z}}{\partial z}\right)=\rho \frac{\partial^{2} u_{r}}{\partial t^{2}} \\
\frac{\partial}{\partial z}\left[C_{11} \frac{\partial u_{z}}{\partial z}+C_{12}\left(\frac{u_{r}}{r}+\frac{\partial u_{r}}{\partial r}\right)\right] \\
+\frac{1}{r} \frac{\partial}{\partial r}\left[C_{66} r\left(\frac{\partial u_{z}}{\partial r}+\frac{\partial u_{r}}{\partial z}\right)\right]=\rho \frac{\partial^{2} u_{z}}{\partial t^{2}} .
\end{gathered}
$$

Equation (6) is recognized as the Navier equation of motion.

As tubes of MWBNNTs are initially concentric and the initial inter-layer distance is approximately equal to the equilibrium spacing, the initial van der Waals interface pressure among any two neighbouring tubes of unloaded MWBNNTs is minor. While exposing the loads, the inter-layer distance exchanges and the van der Waals interface pressure for a point between any two layers alter depending upon the difference of radial deflection. Therefore, if $u_{r}^{k}$ indicates the radial deflection of the layer $k$, the van der Waals interface 
pressures $P_{k(k+1)}$ exposed on the layer $k$ due to the layer $k+1$ is estimated by

$$
P_{k(k+1)}=c_{k}\left(u_{r}^{k+1}-u_{r}^{k}\right),
$$

where $c_{k}$ represents the van der Waals interface coefficient between layer $k$ and layer $k+1$. An analytic rule including the tube-tube potentials is ${ }^{23}$

$$
\Phi\left(R_{k}\right)=-\frac{2 \pi n_{\sigma}^{2} A}{4 R_{0}^{4}}\left[\left(\frac{R_{0}}{R_{k}}\right)^{4}-0.4\left(\frac{R_{0}}{R_{k}}\right)^{10}\right],
$$

where $R$ is the inter-layer distance between tube $k$ and tube $k+1, n_{\sigma}$ the mean surface density of atoms, $A$ the attractive constants in the Lennard-Jones potential and $R_{0}$ the equilibrium distance at the minimum energy for the two interacting entities. Second derivative of equation (8) yields

$$
\frac{\mathrm{d}^{2} \Phi\left(R_{k}\right)}{\mathrm{d} R^{2}}=-\frac{2 \pi n_{\sigma}^{2} A}{4 R_{0}^{4}}\left[20\left(\frac{R_{0}}{R_{k}}\right)^{6}-44\left(\frac{R_{0}}{R_{k}}\right)^{12}\right] .
$$

As the van der Waals interface coefficient $c_{k}$ defined by the second derivative of the energy-inter-layer spacing relation between layer $k+1$ and layer $k$, equation (9) yields

$$
c_{k}(R)=-\eta R_{k}^{-6}+\lambda R_{k}^{-12},
$$

where

$$
\eta=10 \pi n_{\sigma}^{2} A R_{0}^{2}, \quad \lambda=22 \pi n_{\sigma}^{2} A R_{0}^{8} .
$$

In hexagonal mesh of atoms, every atom corresponds to the area of $3 \sqrt{3} a^{2} / 4$, where $a$ is the length between two adjacent atoms. ${ }^{24}$ For BNNTs $a_{B N}=0.144 \mathrm{~nm},{ }^{25}$ hence, mean surface density of atoms is

$$
n_{\sigma}(B-N)=4 / 3 \sqrt{3} a_{B N}^{2}=37.1241 / \mathrm{nm}^{2} .
$$

Substituting $n_{\sigma}$ from equation (12) and $A_{B N}=31.92 \times$ $10^{-6} \mathrm{eV} \mathrm{nm}^{626}$ and $R_{0}=0.344 \mathrm{~nm}^{27}$ in equation (11) yields $\eta=0.262 \mathrm{GPa} \mathrm{nm}^{-1}$ and $\lambda=9.55 \times 10^{-4} \mathrm{GP} \mathrm{nm}^{-1}$.

\section{Finite element formulation}

At this point a two-dimensional finite element model is developed. By employing the Rayleigh-Ritz method to the system of Navier equation in equation (6), weak formulation is established as

$$
\begin{aligned}
\int\left\{\frac{\partial w_{1}}{\partial r}\left[C_{11} r \frac{\partial u_{r}}{\partial r}+C_{12}\left(u_{r}+r \frac{\partial u_{z}}{\partial z}\right)\right]\right. \\
+\frac{\partial w_{1}}{\partial z}\left[C_{66} r\left(\frac{\partial u_{z}}{\partial r}+\frac{\partial u_{r}}{\partial z}\right)\right] \\
\left.+w_{1}\left[C_{11} \frac{u_{r}}{r}+C_{12}\left(\frac{\partial u_{z}}{\partial z}+\frac{\partial u_{r}}{\partial r}\right)\right]+\rho r w_{1} \ddot{u}_{r}\right\} \\
\mathrm{d} r \mathrm{~d} z-\oint w_{1} t_{r} \mathrm{~d} s=0
\end{aligned}
$$

$$
\begin{gathered}
\int\left\{\frac{\partial w_{2}}{\partial z}\left[C_{11} r \frac{\partial u_{z}}{\partial z}+C_{12}\left(u_{r}+r \frac{\partial u_{r}}{\partial r}\right)\right]\right. \\
\left.+\frac{\partial w_{2}}{\partial r}\left[C_{66} r\left(\frac{\partial u_{z}}{\partial r}+\frac{\partial u_{r}}{\partial z}\right)\right]+\rho r w_{2} \ddot{u}_{z}\right\} \\
\mathrm{d} r \mathrm{~d} z-\oint w_{2} t_{z} \mathrm{~d} s=0,
\end{gathered}
$$

where $w_{1}$ and $w_{2}$ are the weight functions and $t_{r}$ and $t_{z}$ the force tractions on the boundary of the MWBNNT, which are determined as

$$
t_{r}=\sigma_{r} n_{r}+\sigma_{r z} n_{z}, \quad t_{z}=\sigma_{z} n_{z}+\sigma_{r z} n_{r},
$$

where $n_{r}$ and $n_{z}$ are the normal vectors in radial and longitudinal directions, respectively. In equation (13), the dot symbolizes the derivative via time. Next, a MWBNNT in which every layer is separated into several rectangular elements which have two degrees of freedom in each node is considered and are placed in the radial and axial directions as shown in figure 2. Also, spring elements are applied between tubes to simulate the van der Waals interactions.

The interpolation functions are chose linearly as

$$
\begin{aligned}
& \psi_{1}=\left(1-\frac{r}{a}\right)\left(1-\frac{z}{b}\right), \quad \psi_{2}=\frac{r}{a}\left(1-\frac{z}{b}\right), \\
& \psi_{3}=\frac{z}{b}\left(1-\frac{r}{a}\right), \quad \psi_{4}=\frac{z}{b} \frac{r}{a} .
\end{aligned}
$$

Also, deflections are estimated with sum of interpolation functions as

$$
u_{r}=\sum_{j=1}^{n_{c}} u_{j} \psi_{j}, \quad u_{z}=\sum_{j=1}^{n_{c}} v_{j} \psi_{j},
$$

where $n_{c}$ is the number of nodes in every element. In the following, the weight functions are substituted by the interpolation functions to obtain the next weak relation

$$
\begin{gathered}
\int\left\{C_{11} r \frac{\partial \psi_{i}}{\partial r} \frac{\partial \psi_{j}}{\partial r} u_{j}+C_{12} \frac{\partial \psi_{i}}{\partial r} \psi_{j} u_{j}+C_{12} r \frac{\partial \psi_{i}}{\partial r} \frac{\partial \psi_{j}}{\partial z} v_{j}\right. \\
+C_{12} \frac{\partial \psi_{j}}{\partial z} \psi_{i} v_{j}+C_{66} r \frac{\partial \psi_{i}}{\partial z} \frac{\partial \psi_{j}}{\partial z} u_{j}+C_{66} r \frac{\partial \psi_{i}}{\partial z} \frac{\partial \psi_{j}}{\partial r} v_{j} \\
\left.+\frac{C_{11}}{r} \psi_{i} \psi_{j} u_{j}+C_{12} \frac{\partial \psi_{j}}{\partial r} \psi_{i} u_{j}+\rho r \psi_{i} \psi_{j} \ddot{u}_{j}\right\} \\
\mathrm{d} r \mathrm{~d} z=\oint \psi_{i} t_{r}, \\
\int\left\{C_{66} r \frac{\partial \psi_{i}}{\partial r} \frac{\partial \psi_{j}}{\partial r} v_{j}+C_{66} r \frac{\partial \psi_{i}}{\partial r} \frac{\partial \psi_{j}}{\partial z} u_{j}+C_{11} r \frac{\partial \psi_{i}}{\partial z} \frac{\partial \psi_{j}}{\partial z} v_{j}\right. \\
\left.+C_{12} \frac{\partial \psi_{i}}{\partial z} \psi_{j} u_{j}+C_{12} r \frac{\partial \psi_{i}}{\partial z} \frac{\partial \psi_{j}}{\partial r} u_{j}+\rho r \psi_{i} \psi_{j} \ddot{v}_{j}\right\} \\
\mathrm{d} r \mathrm{~d} z=\oint \psi_{i} t_{z} \mathrm{~d} s .
\end{gathered}
$$




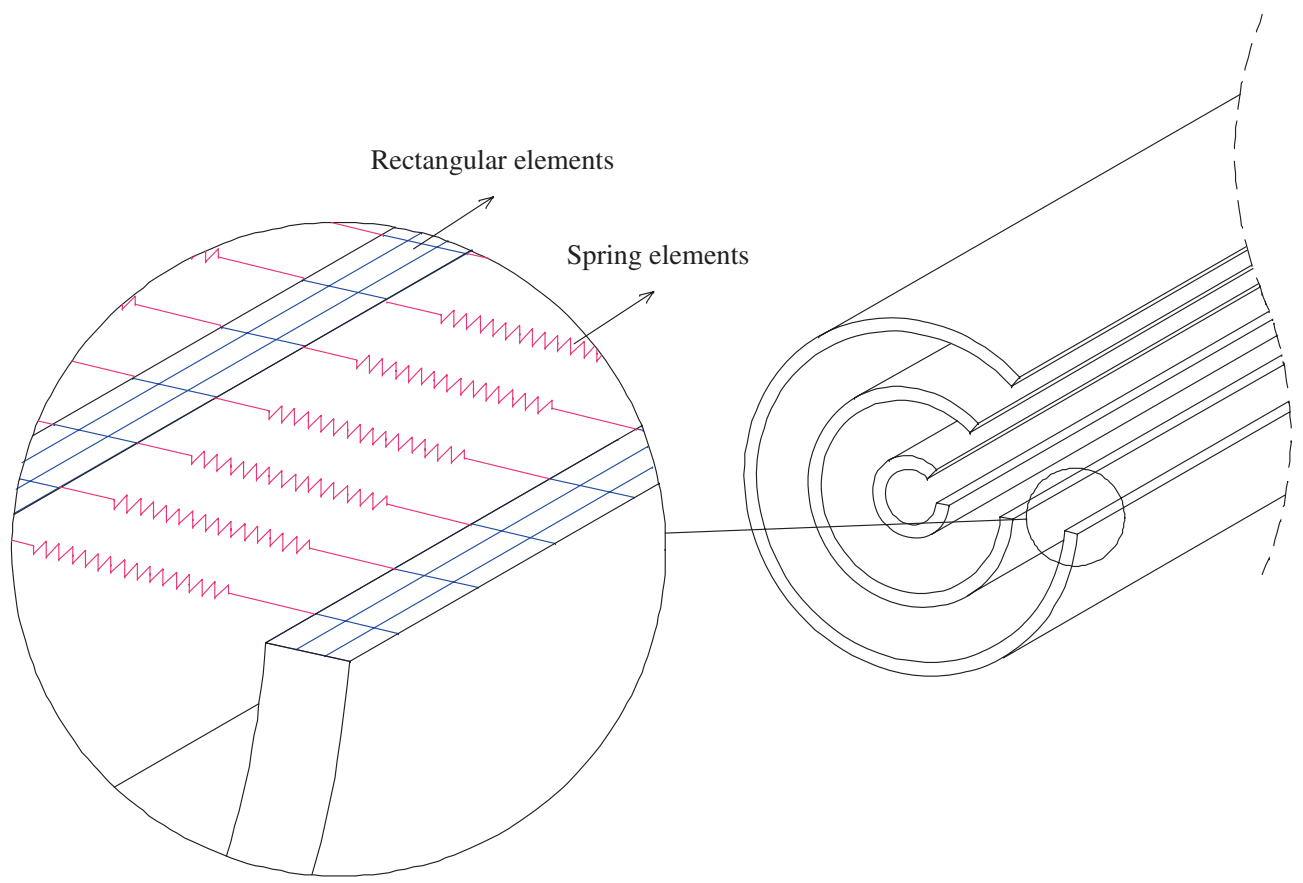

Figure 2. System of elements in the model of MWBNNT.

Equation (17) is demonstrated by matrix form as

$$
\begin{aligned}
& \left.\left[\begin{array}{cc}
{[M]} & {[0]} \\
{[0]} & {[M]}
\end{array}\right]\left\{\begin{array}{l}
\{\ddot{u}\} \\
\{\ddot{v}\}
\end{array}\right\}+\left[\begin{array}{l}
K^{11} \\
\left.K^{21}\right]
\end{array}\right]\left[\begin{array}{l}
K^{12} \\
K^{22}
\end{array}\right]\right\}\left\{\begin{array}{l}
\{u\} \\
\{v\}
\end{array}\right\} \\
& =\left\{\begin{array}{l}
\left\{F^{1}\right\} \\
\left.F^{2}\right\}
\end{array}\right\},
\end{aligned}
$$

where the parts of stiffness and mass matrices and force vectors of every element are determined as

$$
\begin{aligned}
M_{i j}= & \int \rho \psi_{i} \psi_{j} r \mathrm{~d} r \mathrm{~d} z \\
K_{i j}^{11}= & \int\left\{C_{11} r \frac{\partial \psi_{i}}{\partial r} \frac{\partial \psi_{j}}{\partial r}+C_{12} \frac{\partial \psi_{i}}{\partial r} \psi_{j}+\frac{C_{11}}{r} \psi_{i} \psi_{j}\right. \\
& \left.+C_{12} \frac{\partial \psi_{j}}{\partial r} \psi_{i}+C_{66} r \frac{\partial \psi_{i}}{\partial z} \frac{\partial \psi_{j}}{\partial z}\right\} \mathrm{d} r \mathrm{~d} z, \\
K_{i j}^{12}= & K_{j i}^{21}=\int\left\{C_{12} r \frac{\partial \psi_{i}}{\partial r} \frac{\partial \psi_{j}}{\partial z}+C_{12} \psi_{i} \frac{\partial \psi_{j}}{\partial z}\right. \\
& \left.+C_{66} r \frac{\partial \psi_{i}}{\partial z} \frac{\partial \psi_{j}}{\partial r}\right\} \mathrm{d} r \mathrm{~d} z, \\
K_{i j}^{22}= & \int\left\{C_{66} r \frac{\partial \psi_{i}}{\partial r} \frac{\partial \psi_{j}}{\partial r}+C_{11} r \frac{\partial \psi_{i}}{\partial z} \frac{\partial \psi_{j}}{\partial z}\right\} \mathrm{d} r \mathrm{~d} z, \\
F_{i}^{1}= & \oint \psi_{i} t_{r} \mathrm{~d} s, \\
F_{i}^{2}= & \oint \psi_{i} t_{z} \mathrm{~d} s .
\end{aligned}
$$

Here, all of the integrations in equation (3) are computed analytically through every element area to keep away from any numerical errors. The matrices $[K]$ and $[M]$ are computed for every element particularly and subsequently via an assemblage procedure, the global matrices for the whole MWBNNT are established. Thus, the global form of equation (18) can be presented as

$$
[M]^{\mathrm{a}}\{\ddot{U}\}+[K]^{\mathrm{a}}\{U\}=\{F\}^{\mathrm{a}},
$$

whereas $[K]^{\mathrm{a}},[M]^{\mathrm{a}}$ and $\{F\}^{\mathrm{a}}$ are the assembled stiffness, mass and force matrices, respectively. Here, the Newmark direct integration technique is employed to solve equation (20) and finding transient responses.

\section{Numerical results and discussions}

In this study, properties of BNNT are selected as in-plane moduli of elasticity $E_{\mathrm{S}}=0.271 \mathrm{TPa} \mathrm{nm}, v=0.211,{ }^{28}$ and the mass density $\rho=2.4 \mathrm{~kg} \mathrm{~m}^{-3} .{ }^{29}$ Thickness of MWBNNT is estimated as $h_{m}=r_{\text {out }}-r_{\text {in }}$ where $r_{\text {in }}$ and $r_{\text {out }}$ are the radius of inmost and outmost layers, respectively. A SWBNNT is determined from a pair $(m, n)$, which is addressed as the chirality of SWBNNT. The $(n, n)$ and $(n, 0)$ BNNTs are named armchair and zigzag BNNTs, respectively, and the others are called chiral BNNTs. The radius $r$ of a tube of MWBNNTs is determined by its bond length $a_{0}$ and employing the rule 25

$$
r=\frac{a_{0} \sqrt{3}}{2 \pi} \sqrt{\left(m^{2}+n^{2}+m n\right)}, \quad a_{0}=1.44 \AA .
$$


For validation of the method dependency of the radial breathing mode frequency with the nanotube radius is studied. This dependency is shown by many investigators with the relation

$$
\omega_{\mathrm{RBM}}=\frac{A}{r} \mathrm{~cm}^{-1},
$$

where $r$ is the nanotube radius. The values of $A$ for BNNTs are found to be $101.08,{ }^{29} 102.5^{30}$ and $99 .{ }^{31}$ Here, this measure is obtained as $102.11 \mathrm{~cm}^{-1} \mathrm{~nm}$, which has a good agreement with those found by the other investigators.

In the next calculations according to inmost radius to thickness ratio $\lambda$, three models of MWBNNTs are regarded and listed in table 1 that sorted as bulky MWBNNT $(\lambda=1)$ and thin MWBNNT $(\lambda=7)$. It is noted that models 1 and 2 have the same outmost radius and models 2 and 3 have identical thickness. For all MWBNNTs, length-to-outmost radius ratio is considered equal to 100 . In the next examples, MWBNNTs that exposed to impacts of external pressure or initial longitudinal deflection are studied and the dynamical reactions are determined.

\subsection{MWBNNTs subjected to external pressure impact}

Here, external pressure of $100 \mathrm{MPa}$ is exposed abruptly to the entire outmost wall of MWBNNTs. Variations of circumferential stress $v s$. time for middle points of three layers of every aforementioned model of MWBNNTs are demonstrated in figure 3 . It is revealed that the circumferential stresses of all tubes of thin MWBNNT (model 2) are approximately identical but they are different at every tube of bulky MWBNNTs (models 1 and 3) that the outmost layer has the maximum stress. Moreover, the maximum stress of outmost layer of model 2 (3-layer MWBNNT) is greater than that of outmost layer of model 1 (9-layer MWBNNT), while their outmost layers have identical radius. Therefore, for external pressure impact in a MWBNNT with fixed outmost tube radius, its maximum circumferential stress reduces as the number of tubes raises. Also, in inner tubes of MWBNNTs which subjected to external pressure impact, tensile circumferential stresses are created. The ratio of tensile per compressive in maximum values of circumferential stress for models 1,2 and 3 of MWBNNTs are 0.728, 0.132 and 0.206, respectively. Hence, in bulky MWBNNTs with various layers which are subjected to external pressure impact, major tensile circumferential stress is generated in inmost layer.

Table 1. Geometrical information for three models of MWBNNTs.

\begin{tabular}{lccc}
\hline Model & 1 & 2 & 3 \\
\hline Number of layers & 9 & 3 & 3 \\
Innermost radius $r_{\text {in }}(\mathrm{nm})$ & 2.7502 & 4.8125 & 0.6875 \\
Thickness $h_{m}(\mathrm{~nm})$ & 2.7502 & 0.6875 & 0.6875 \\
Innermost radius-to-thickness & 1 & 7 & 1 \\
$\quad$ ratio $\lambda=r_{\text {in }} / h_{m}$ & & & \\
\hline
\end{tabular}

It is intended here to find the frequencies of oscillations for outmost layer of MWBNNTs which subjected to external pressure impact. It can be made by measuring the time difference between the first and last picks from the diagrams of time history of circumferential stress. Computed frequencies with this process are $24.02,19.98$ and $88.31 \mathrm{~cm}^{-1}$, respectively, for models 1, 2 and 3 of MWBNNTs. Therefore, in MWBNNTs with identical outmost radius, radial oscillations frequency enhances by the increment in the amount of layers. However, in MWBNNTs with identical thickness, radial oscillations frequency enhances by the reduction in inmost radius.

In MWBNNTs which is subjected to external pressure impact, extensive tensile waves are also created. To examine these, model 3 of MWBNNTs is regarded and variations of axial deflection $v s$. time for point $z=l / 4$ on outmost tube are demonstrated in figure 4 . It is seen that the value of axial deflection remains invariant before the wave gets to that position which is reasonable by the assumption that the wave commences from MWBNNT edges. When the tensile wave reaches the mentioned position, its longitudinal deflection is varied linearly until alternate tensile wave reaches to this position from the other end of MWBNNT. As well, reverse deflections happen subsequently by turning of the waves from edges of MWBNNT. For better examination of these processes, figure 5 shows variations of axial strain and stress $v s$. time for point $z=l / 4$ on outmost tube of model 3 of MWBNNTs which is subjected to external pressure impact. As seen, once the longitudinal wave from one edge of tube reaches to that position, the values of longitudinal strain and stress vary abruptly. Besides, axial stress forever oscillates according to the variations in radial deflections. As seen from figure 5b, before the arrival of the longitudinal wave to the mentioned position, the mean longitudinal stress is compressive. Therefore, the longitudinal wave reaching this position causes a decrease in the amount of longitudinal stresses and the mean longitudinal stress becomes nearly zero. Linking the alternate longitudinal wave from other edge of MWBNNT to this position makes an increment in the amounts of longitudinal stress while the mean longitudinal stress becomes tensile. This treatment of longitudinal stress is similar for the other models of MWBNNTs. The ratios of highest longitudinal stresses per external pressure are 7.64, 11.87 and 4.23, respectively, for models 1, 2 and 3 of MWBNNTs. Hence, longitudinal stresses are significant and must be evaluated for MWBNNTs which are subjected to external pressure impact.

At present, dynamical treatment of MWBNNTs under partially loading is studied which only a half length of outer surface of MWBNNTs are exposed to impact of $100 \mathrm{MPa}$ external pressure. This condition may occur in some situations such as nanocomposites or nanosensors. Distributions of radial deflection along the longitudinal direction at a moment of time ( $2 \mathrm{ps}$ ) for three models of MWBNNTs are illustrated in figure 6. It is seen that the unloaded parts of MWBNNTs are affected by the deflections of their loaded parts, as much as about $10 \%$ of the maximum deflections 


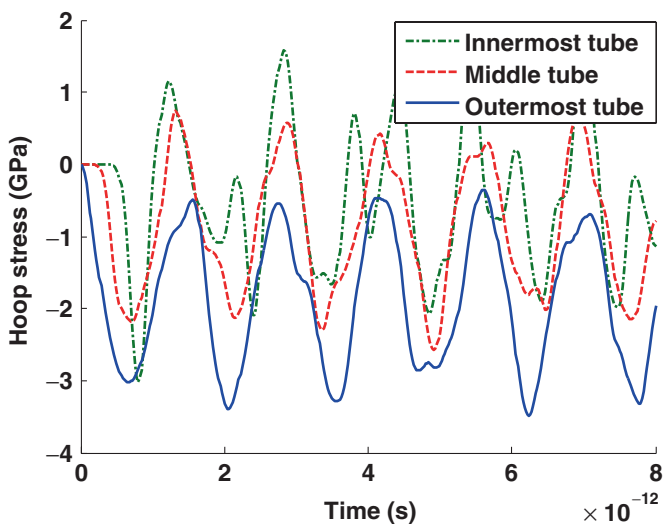

(a)

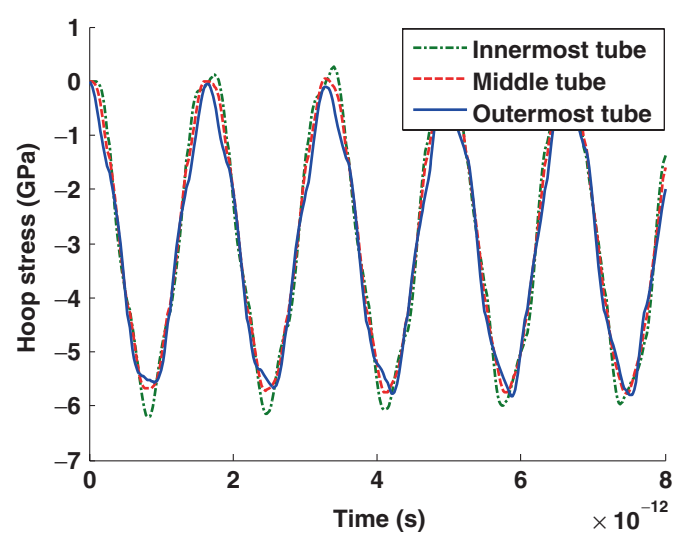

(b)

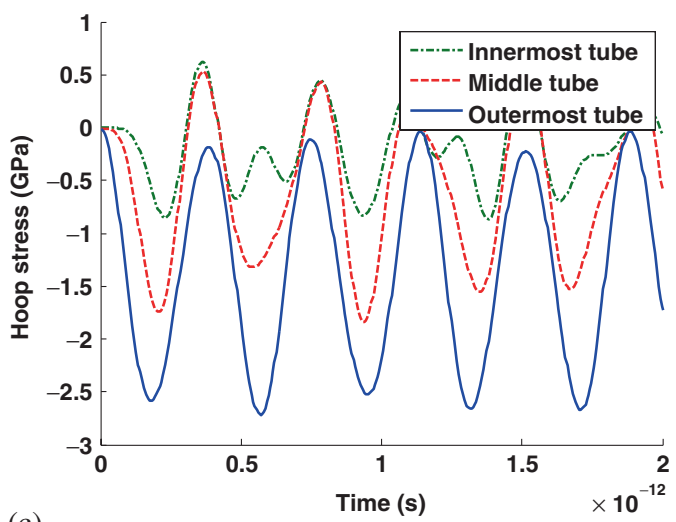

(c)

Figure 3. Variations of circumferential stress vs. time for models (a) 1, (b) 2 and (c) 3 of MWBNNTs which is subjected to external pressure impact.

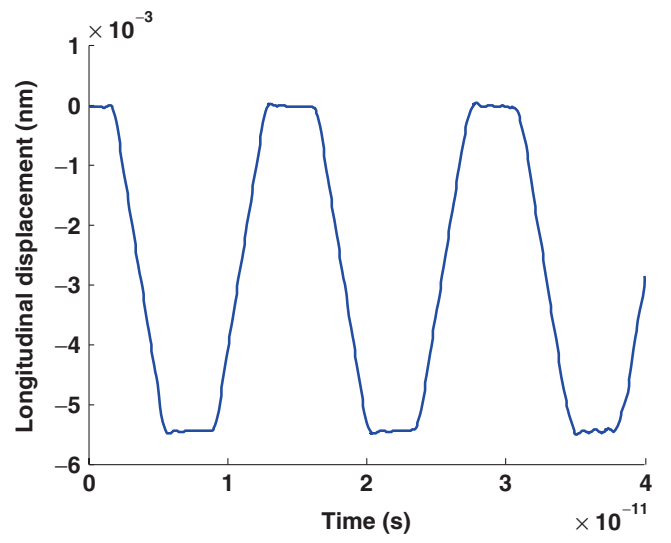

Figure 4. Variations of axial deflection vs. time for model 3 of MWBNNTs under external pressure impact.

of MWBNNTs. Also, for models 1 and 2 of MWBNNTs, amplitude of oscillations for every tube of thin MWBNNT is greater than those of bulky MWBNNT with identical outmost radius. Hence, for an external pressure impact, the van der Waals interactions moderate maximum displacements of bulky MWBNNTs in comparison with thin MWBNNTs. Also, by evaluating model 1 with model 3 (bulky MWBNNTs with dissimilar radii), it is revealed that the ratio of inmost layer deflection per outmost layer deflection, for 3layer MWBNNT is lower than that of 9-layer MWBNNT. That is, the van der Waals interactions have more beneficial shift impact wave in large radius tubes than in small radius tubes. Finally, comparison among models 2 and 3 shows that even for MWBNNTs with identical thickness, all tubes of thin MWBNNT oscillate similarly and with identical deflection but in bulky MWBNNT, defection of vibrations for outer tubes are higher than those of inner tubes. Therefore, inner tubes of thin MWBNNTs are more influenced from external impact than those of bulky MWBNNTs. By these explanations and investigating figure 6 , it is seen that in a bulky MWBNNTs which is subjected to external pressure impact, the influences of van der Waals interactions are feeble and the absolute amount of mean deflection for inner layers are decreased by the reducing of tubes' radius.

\subsection{MWBNNTs subjected to impact of initial axial deflection}

Here, one tenth of length of all tubes of MWBNNTs are subjected to an abrupt initial longitudinal deflection equal to $0.01 \mathrm{~nm}$. Besides, other initial and boundary conditions are presumed unchanged. For this impact loading, longitudinal wave produces and spreads through the length of 


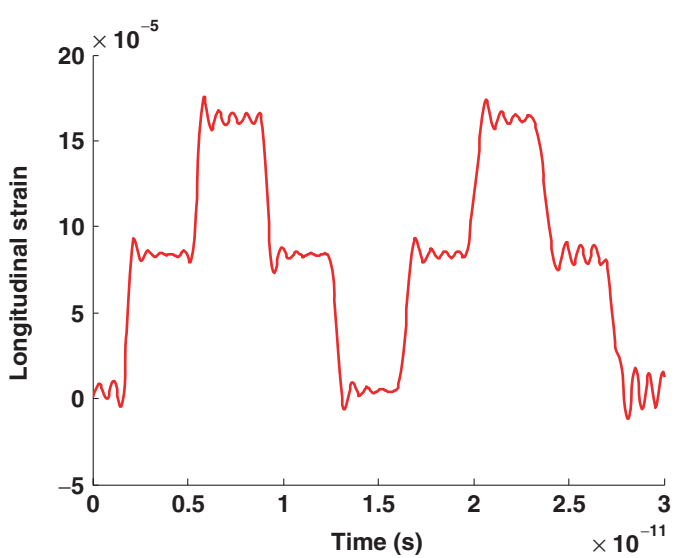

(a)

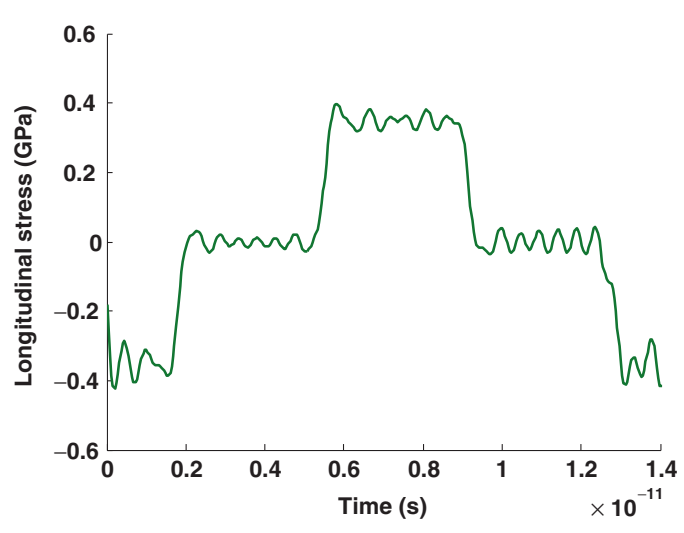

(b)

Figure 5. Variations of longitudinal (a) strain and (b) stress $v s$. time for model 3 of MWBNNTs which is subjected to external pressure impact.

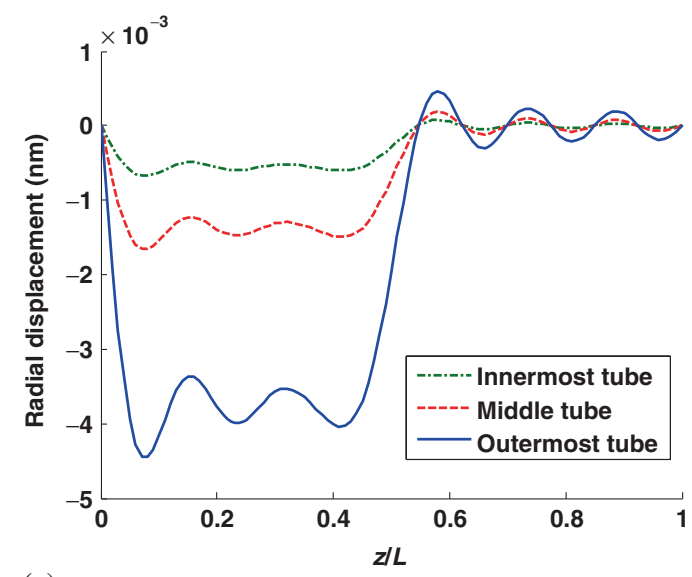

(a)

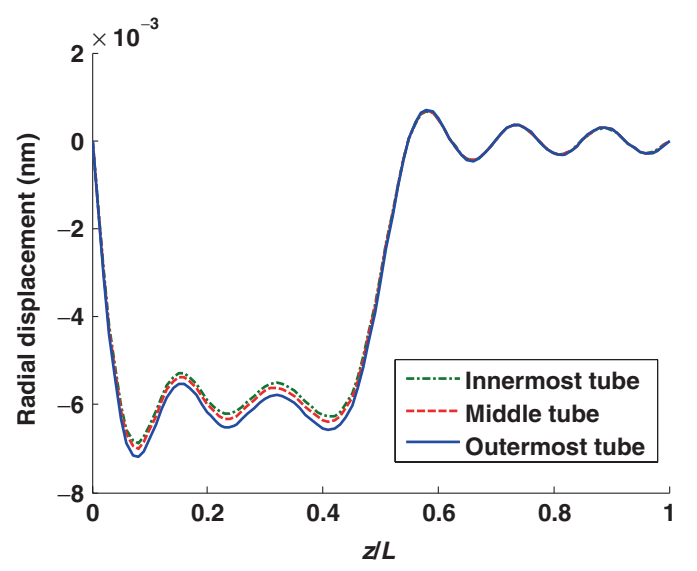

(b)

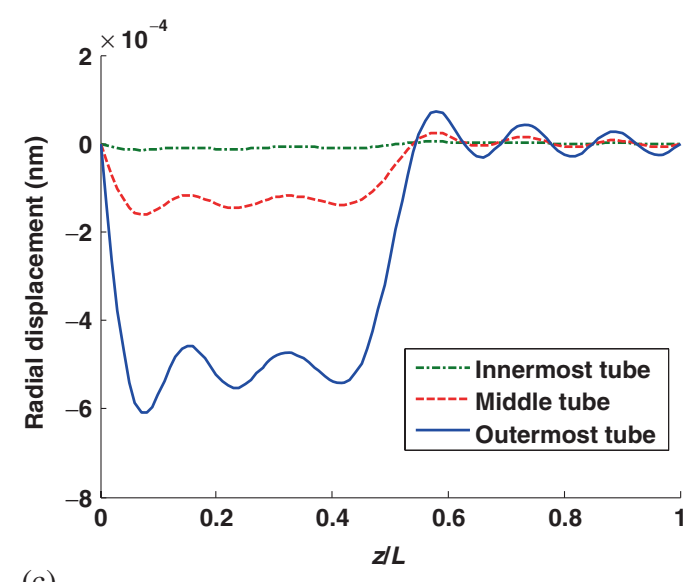

(c)

Figure 6. Distribution of radial deflection along the longitudinal direction for models (a) 1, (b) 2 and (c) 3 of MWBNNTs under partially external pressure impact.

MWBNNTs. Variations of longitudinal deflection and stress vs. time for the middle position of outmost layer of model 2 of MWBNNTs are illustrated in figure 7. It is found that while the longitudinal wave reaches the mentioned position, the amount of axial deflection and stress exchange suddenly and the diagrams have two jumps. The first jump is related 


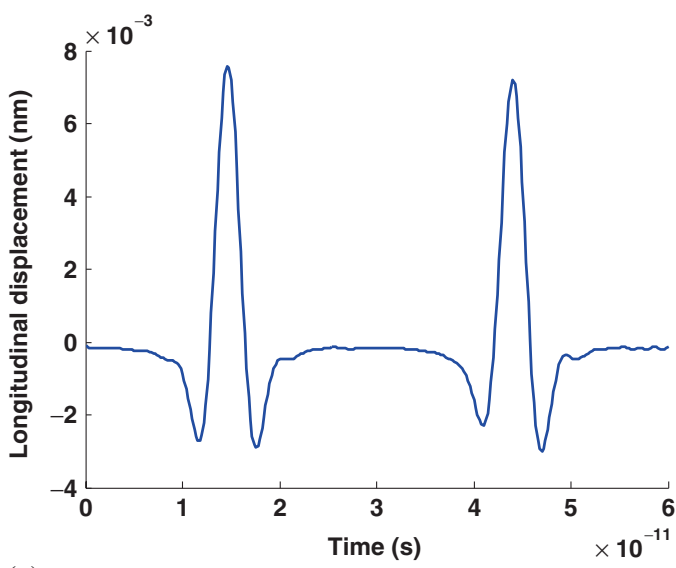

(a)

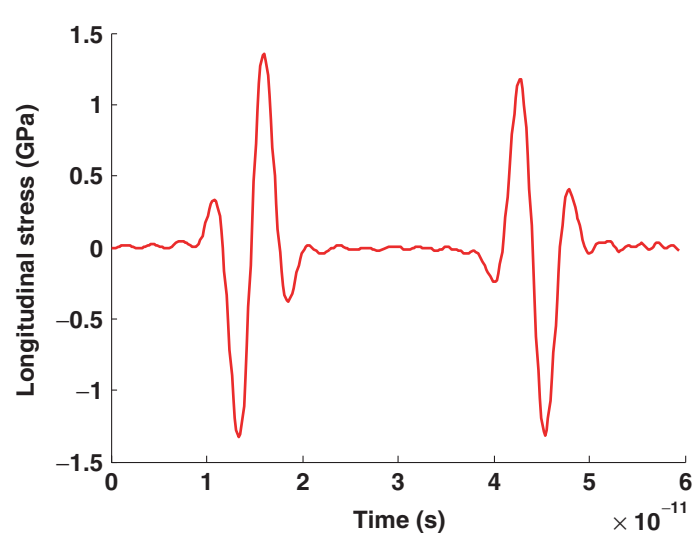

(b)

Figure 7. Variations of axial (a) deflection and (b) stress for model 2 of MWBNNTs with impact of initial axial deflection.

Table 2. Maximum longitudinal stress for three models of MWBNNTs (GPa).

\begin{tabular}{lccc}
\hline Models & 1 & 2 & 3 \\
\hline Innermost tube & 1.4862 & 1.4387 & 5.4553 \\
Middle tube & 1.4683 & 1.4875 & 5.4928 \\
Outmost tube & 1.4763 & 1.4750 & 5.4826 \\
\hline
\end{tabular}

to spread of wave and the second jump is related to reflex of the wave. Hence, the longitudinal wave moves one length of MWBNNT in time between jump to jump and by computing this time, the wave speed can be found. This process is like for other models of MWBNNTs and so, the wave speeds for outmost layer of models 1, 2 and 3 of MWBNNTs are $18.4751,18.5673$ and $18.3867\left(\mathrm{~km} \mathrm{~s}^{-1}\right)$, respectively. It is revealed that the longitudinal wave speed is approximately identical for outmost layer of all models of MWBNNTs and thus it is free from tubes' radius.

However, there is different treatment on maximum longitudinal stresses produced in MWBNNTs which is subjected to longitudinal deflection impact. For a better study, maximum longitudinal stresses of three models of MWBNNTs are shown in table 2. It is observed that the maximum values of longitudinal stresses for all tubes of every MWBNNT are approximately identical but they are different for MWBNNTs with unlike outmost tubes radius. Therefore, by raising the outmost tube radius of MWBNNT under axial deflection impact, the maximum value of longitudinal stresses reduces.

\section{Conclusions}

Dynamic treatments of MWBNNTs with finite length are analysed using the continuum multiple elastic shell model. By formulating the finite element model, dynamical reactions are studied for MWBNNTs which are subjected to impacts of external pressure or initial axial deflection.
Besides, influences of size are studied by considering three models of MWBNNTs with 3 and 9 tubes and different outmost radii. The results show that, when external pressure impact exposed to a MWBNNT with fixed outmost layer radius, by enhancing the number of tubes, the maximum of circumferential stress reduces. On the other hand, maximum deflection for every tube of thin MWBNNT is bigger than those of bulky MWBNNT with identical outmost radius. Also, in the case of partially loading, it is seen that the unloaded parts of MWBNNTs are affected by the deflections of their loaded parts, as much as about $10 \%$ of the maximum deflections of MWBNNTs. Finally, in MWBNNTs with impact of initial axial deflection, the longitudinal wave speed is not dependent on tubes' radius but by enhancing the outmost tube radius, the maximum longitudinal stress reduces.

\section{Acknowledgement}

We are grateful to Islamic Azad University of Neyshabur for supporting this work.

\section{References}

1. Griebel M, Hamaekers J and Heber F 2009 Comput. Mater. Sci. 451097

2. Jiang L and Guo W 2011 J. Mech. Phys. Solids 591204

3. Oh E-S 2010 Mater. Lett. 64859

4. Vahedi Fakhrabad D and Shahtahmassebi N 2013 Mater. Chem. Phys. 138963

5. Shen H 2009 Comput. Mater. Sci. 47220

6. Ghorbanpour Arani A and Amir S 2013 Physica B: Condens. Matter 4191

7. Mosallaie Barzoki A A, Ghorbanpour Arani A, Kolahchi R and Mozdianfard M R 2012 Appl. Math. Model. 362983

8. Ghorbanpour Arani A, Amir S, Shajari A R and Mozdianfard M R 2012 Composites Part B: Engineering 43195 
9. Ghorbanpour Arani A, Shajari A R, Amir S and Loghman A 2012 Physica E: Low-dimensional Systems Nanostruc. 45109

10. Ghorbanpour Arani A, Abdollahian M, Kolahchi R and Rahmati A H 2013 Composites Part B: Engineering 51291

11. Talebian S T, Tahani M, Hosseini S M and Abolbashari M H 2010 Comput. Mater. Sci. 49283

12. Talebian S T, Tahani M, Abolbashari M H and Hosseini S 2011 Arch. Appl. Mech. 811129

13. Talebian S T, Tahani M, Abolbashari M H and Hosseini S M 2012 Comput. Mater. Sci. 61291

14. Talebian S T, Tahani M, Abolbashari M H and Hosseini S 2012 Acta Mech. Sin. $\mathbf{2 8} 720$

15. Panchal M B and Upadhyay S H 2013 Physica E: Lowdimensional Systems Nanostruct. 5073

16. Zhang S, Liu Y, Xia M, Zhang L, Zhang E, Liang R and Zhao S 2008 Physica B: Condens. Matter 4034196

17. Ghorbanpour Arani A, Kolahchi R and Khoddami Maraghi Z 2013 Appl. Math. Model. 377685

18. Ghorbanpour Arani A and Roudbari M A 2013 Thin Solid Films 542232

19. Khodami Maraghi Z, Ghorbanpour Arani A, Kolahchi R, Amir S and Bagheri M R 2013 Composites Part B: Engineering 45 423
20. Ghorbanpour Arani A, Hashemian M and Kolahchi R 2013 Composites Part B: Engineering $\mathbf{5 4} 298$

21. Panchal M B, Upadhyay S H and Harsha S P 2013 Sens. Actuators A: Physical 197111

22. Boresi A P, Chong K P and Lee J D 2010 Elasticity in engineering mechanics (New York: John Wiley \& Sons, Inc.) p 455

23. Girifalco L A, Hodak M and Lee R S 2000 Phys. Rev. BCondens. Matter Mater. Phys. 6213104

24. He X Q, Kitipornchai S and Liew K M 2005 J. Mech. Phys. Solids $\mathbf{5 3} 303$

25. Oku T and Narita I 2004 Diam. Relat. Mater. 131254

26. Thamwattana N and Hill J M 2009 Physica B 4043906

27. Zhi C, Bando Y, Tang C and Golberg D 2010 Mater. Sci. Eng. R: Rep. 7092

28. Kudin K N, Scuseria G E and Yakobson B I 2001 Phys. Rev. B 64235406

29. Velasco V R and Muñoz M C 2009 Surf. Sci. 6032950

30. Akdim B, Pachter R, Duan X and Adams W W 2003 Phys. Rev. B 67245404

31. Fakrach B, Rahmani A, Chadli H, Bentaleb M, Sbai K, Benhamou M, Bantignies J L and Sauvajol J L 2013 Physica E: Low-dimensional Systems Nanostruct. 48140 